\title{
Sufficient conditions to solve two systems of integral equations via fixed point results
}

\author{
Tahair Rasham ${ }^{1 *} \mathbb{D}$, Abdullah Shoaib², Giuseppe Marino ${ }^{3}$, Badriah A.S. Alamrit and Muhammad Arshad ${ }^{1}$
}

\author{
"Correspondence: \\ tahir_resham@yahoo.com \\ 'Department of Mathematics and \\ Statistics, International Islamic \\ University, Islamabad, Pakistan \\ Full list of author information is \\ available at the end of the article
}

\begin{abstract}
The purpose of this paper is to study the solution of two systems of nonlinear integral equations via fixed point results in a complete dislocated $b$-metric space. Also the notion of graphic contractions on a closed set for two families of graph dominated multivalued mappings is introduced. Our results generalize some previous results in the existing literature.
\end{abstract}

MSC: $46540 ; 47 \mathrm{H} 10 ; 54 \mathrm{H} 25$

Keywords: Fixed point; Two families of multivalued mapping; Dislocated $b$-metric space; Application to the system of nonlinear integral equations

\section{Introduction and preliminaries}

Fixed point theory plays a fundamental role in functional analysis. Nadler [18] started the investigation of fixed point results for the set-valued functions. Due to its significance, a large number of authors have proved many interesting multiplications of his result (see [1-4, 6, 8-29]).

Nazir et al. [19] showed common fixed point results for the family of generalized multivalued $F$-contraction mappings in ordered metric spaces. Recently Shoaib et al. [27] discussed some theorems for a family of set-valued functions. Rasham et al. [22] proved multivalued fixed point theorems for new $F$-contractive functions on dislocated metric spaces.

In this paper, we have obtained a common fixed point of two families of multivalued mappings satisfying generalized rational type $\alpha_{*}-\psi$-dominated contractive conditions on a closed set in a complete dislocated $b$-metric space. We have used a weaker class of strictly increasing mappings $A$ rather than the class of mappings $F$ used by Wardowski [29]. Examples have been given to demonstrate the variety of our results. Moreover, we investigate our results in a better framework of dislocated $b$-metric space. New results in ordered spaces, partial $b$-metric space, dislocated metric space, partial metric space, $b$ metric space, and metric space can be obtained as corollaries of our results. We give the following concepts which will be helpful to understand the paper.

Definition 1.1 ([14]) Let $M$ be a nonempty set, and let $d_{b}: M \times M \rightarrow[0, \infty)$ be a function. If, for any $x, y, z \in M$, the following conditions hold:

(i) $d_{b}(x, y) \leq b\left[d_{b}(x, z)+d_{b}(z, y)\right]$, (where $b \geq 1$ ).

(ii) $d_{b}(x, y)=0$ implies $x=y$;

(c) The Author(s) 2019. This article is distributed under the terms of the Creative Commons Attribution 4.0 International License (http://creativecommons.org/licenses/by/4.0/), which permits unrestricted use, distribution, and reproduction in any medium, provided you give appropriate credit to the original author(s) and the source, provide a link to the Creative Commons license, and indicate if changes were made. 
(iii) $d_{b}(x, y)=d_{b}(y, x)$.

Then $d_{b}$ is called a dislocated $b$-metric with coefficient $b$ (or simply $d_{b}$-metric) and the pair $\left(M, d_{b}\right)$ is called a dislocated $b$-metric space. It should be noted that every dislocated metric is a dislocated $b$-metric with $b=1$.

Note that, if $x=y$, then $d_{b}(x, y)$ may not be 0 . For $x \in M$ and $\varepsilon>0, \overline{B(x, \varepsilon)}=\{y \in M$ : $\left.d_{b}(x, y) \leq \varepsilon\right\}$ is a closed ball in $\left(M, d_{b}\right)$. We use a D.B.M space instead of a dislocated $b$ metric space.

Definition $1.2([14])$ Let $\left(M, d_{b}\right)$ be a D.B.M space.

(i) A sequence $\left\{x_{n}\right\}$ in $\left(M, d_{b}\right)$ is called Cauchy sequence if, given $\varepsilon>0$, there corresponds $n_{0} \in N$ such that for all $n, m \geq n_{0}$ we have $d_{b}\left(x_{m}, x_{n}\right)<\varepsilon$ or $\lim _{n, m \rightarrow \infty} d_{b}\left(x_{n}, x_{m}\right)=0$.

(ii) A sequence $\left\{x_{n}\right\}$ dislocated $b$-converges (for short $d_{b}$-converges) to $x$ if $\lim _{n \rightarrow \infty} d_{b}\left(x_{n}, x\right)=0$. In this case $x$ is called a $d_{b}$-limit of $\left\{x_{n}\right\}$.

(iii) $\left(M, d_{b}\right)$ is called complete if every Cauchy sequence in $M$ converges to a point $x \in M$ such that $d_{b}(x, x)=0$.

Definition 1.3 Let $K$ be a nonempty subset of the D.B.M space $M$, and let $x \in M$. An element $y_{0} \in K$ is called a best approximation in $K$ if

$$
d_{b}(x, K)=d_{b}\left(x, y_{0}\right), \quad \text { where } d_{b}(x, K)=\inf _{y \in K} d_{b}(x, y)
$$

If each $x \in M$ has at least one best approximation in $K$, then $K$ is called a proximinal set. Let $\Psi_{b}$, where $b$ is the coefficient of the D.B.M space $M$. Denote the family of all nondecreasing functions $\psi_{b}:[0,+\infty) \rightarrow[0,+\infty)$ such that $\sum_{k=1}^{+\infty} b^{k} \psi_{b}^{k}(t)<+\infty$ and $b \psi_{b}(t)<t$ for all $t>0$, where $\psi_{b}^{k}$ is the $k$ th iterate of $\psi_{b}$. Also $b^{n+1} \psi_{b}^{n+1}(t)=b^{n} b \psi_{b}\left(\psi_{b}^{n}(t)\right)<b^{n} \psi_{b}^{n}(t)$. We denote $P(M)$ to be the set of all closed proximinal subsets of $M$.

Definition $1.4([28])$ The function $H_{d_{b}}: P(M) \times P(M) \rightarrow R^{+}$, defined by

$$
H_{d_{b}}(N, R)=\max \left\{\sup _{n \in N} d_{b}(n, R), \sup _{r \in R} d_{b}(N, r)\right\}
$$

is called dislocated Hausdorff $b$-metric on $P(M)$.

Definition 1.5 Let $\left(M, d_{b}\right)$ be a D.B.M space. Let $S: M \rightarrow P(M)$ be a multivalued mapping, $\alpha: M \times M \rightarrow[0,+\infty)$ and $\alpha_{*}(i, S i)=\inf \{\alpha(i, l): l \in S i\}$. Let $H \subseteq M$, then $S$ is said to be $\alpha_{*}-$ dominated on $H$, whenever $\alpha_{*}(i, S i) \geq 1$ for all $i \in H$. If $H=M$, then we say that the $S$ is $\alpha_{*}$-dominated. If $S: M \rightarrow M$ is a self mapping, then $S$ is $\alpha$-dominated on $H$, whenever $\alpha(i, S i) \geq 1$ for all $i \in H$.

Lemma 1.6 ([17]) Let $\left(Z, d_{b}\right)$ be a D.B.M space. Let $\left(P(Z), H_{d_{b}}\right)$ be a dislocated Hausdorffbmetric space on $P(Z)$. For all $G, H$ in $P(Z)$ and for any $g \in G$, let $h_{g} \in H$ such that $d_{b}(g, H)=$ $d_{b}\left(g, h_{g}\right)$. Then $H_{d_{b}}(G, H) \geq d_{b}\left(g, h_{g}\right)$ holds. 


\section{Main result}

Let $\left(M, d_{b}\right)$ be a D.B.M space, $c_{0} \in M$, let $\left\{S_{\sigma}: \sigma \in \Omega\right\}$ and $\left\{T_{\beta}: \beta \in \Phi\right\}$ be two families of multifunctions from $M$ to $P(M)$. Let $a \in \Omega$ and $c_{1} \in S_{a} c_{0}$ be an element such that $d_{b}\left(c_{0}, S_{a} c_{0}\right)=d_{b}\left(c_{0}, c_{1}\right)$. Let $c_{2} \in T_{z} c_{1}$ be such that $d_{b}\left(c_{1}, T_{z} c_{1}\right)=d_{b}\left(c_{1}, c_{2}\right)$ where $z \in \Phi$. Let $y \in \Omega$ and $c_{3} \in S_{y} c_{2}$ be such that $d_{b}\left(c_{2}, S_{y} c_{2}\right)=d_{b}\left(c_{2}, c_{3}\right)$. In this way, we get a sequence $\left\{T_{\beta} S_{\sigma}\left(c_{n}\right)\right\}$ in $M$, where $c_{2 n+1} \in S_{i} c_{2 n}, c_{2 n+2} \in T_{j} c_{2 n+1}, n \in \mathbb{N}, i \in \Omega$, and $j \in \Phi$. Also $d_{b}\left(c_{2 n}, S_{i} c_{2 n}\right)=d_{b}\left(c_{2 n}, c_{2 n+1}\right), d_{b}\left(c_{2 n+1}, T_{j} c_{2 n+1}\right)=d_{b}\left(c_{2 n+1}, c_{2 n+2}\right) .\left\{T_{\beta} S_{\sigma}\left(c_{n}\right)\right\}$ is said to be a sequence in $M$ generated by $c_{0}$. If $\left\{S_{\sigma}: \sigma \in \Omega\right\}=\left\{T_{\beta}: \beta \in \Phi\right\}$, then we say $\left\{M S_{\sigma}\left(c_{n}\right)\right\}$ instead of $\left\{T_{\beta} S_{\sigma}\left(c_{n}\right)\right\}$. For $u, v \in M, a>0$, we define $D_{(\sigma, \beta)}(u, v)$ as

$$
D_{(\sigma, \beta)}(u, v)=\max \left\{d_{b}(u, v), \frac{d_{b}\left(u, S_{\sigma} u\right) \cdot d_{b}\left(v, T_{\beta} v\right)}{a+d_{b}(u, v)}, d_{b}\left(u, S_{\sigma} u\right), d_{b}\left(v, T_{\beta} v\right)\right\} .
$$

Theorem 2.1 Let $\left(M, d_{b}\right)$ be a complete D.B.M space. Suppose that there exists a function $\alpha: M \times M \rightarrow[0, \infty)$. Let $r>0, c_{0} \in \overline{B_{d_{b}}\left(c_{0}, r\right)}, A: \mathbb{R}_{+} \rightarrow \mathbb{R}$ be a strictly increasing function and $\left\{S_{\sigma}: \sigma \in \Omega\right\},\left\{T_{\beta}: \beta \in \Phi\right\}$ be two families of $\alpha_{*}$-dominated multivalued mappings from $M$ to $P(M)$ on $\overline{B_{d_{b}}\left(c_{0}, r\right)}$. Suppose that, for some $\psi_{b} \in \Psi_{b}$, there exists $\tau>0$ such that the following holds:

$$
\tau+A\left(H_{d_{b}}\left(S_{\sigma} e, T_{\beta} y\right)\right) \leq A\left(\psi_{b}\left(D_{(\sigma, \beta)}(e, y)\right)\right)
$$

for all $e, y \in \overline{B_{d_{b}}\left(c_{0}, r\right)} \cap\left\{T_{\beta} S_{\sigma}\left(c_{n}\right)\right\}, \alpha(e, y) \geq 1, \sigma \in \Omega, \beta \in \Phi$, and $H_{d_{b}}\left(S_{\sigma} e, T_{\beta} y\right)>0$. Also

$$
\sum_{i=0}^{n} b^{i+1}\left\{\psi_{b}^{i}\left(d_{b}\left(c_{0}, S_{a} c_{0}\right)\right)\right\} \leq r \quad \text { for all } n \in \mathbb{N} \cup\{0\}
$$

Then $\left\{T_{\beta} S_{\sigma}\left(c_{n}\right)\right\}$ is a sequence in $\overline{B_{d_{b}}\left(c_{0}, r\right)}, \alpha\left(c_{n}, c_{n+1}\right) \geq 1$ for all $n \in \mathbb{N} \cup\{0\}$ and $\left\{T_{\beta} S_{\sigma}\left(c_{n}\right)\right\} \rightarrow u \in \overline{B_{d_{b}}\left(c_{0}, r\right)}$. Also, if inequality (2.1) holds, if $\overline{B_{d_{b}}\left(c_{0}, r\right)}$ is a closed set for $e, y \in\{u\}$ and either $\alpha\left(c_{n}, u\right) \geq 1$ or $\alpha\left(u, c_{n}\right) \geq 1$ for all $n \in \mathbb{N} \cup\{0\}$, then $S_{\sigma}$ and $T_{\beta}$ have a common fixed point $u$ in $\overline{B_{d_{b}}\left(c_{0}, r\right)}$ for all $\sigma \in \Omega$ and $\beta \in \Phi$.

Proof Consider a sequence $\left\{T_{\beta} S_{\sigma}\left(c_{n}\right)\right\}$. From (2.2), we get

$$
d_{b}\left(c_{0}, c_{1}\right) \leq b d_{b}\left(c_{0}, S_{a} c_{0}\right)<\sum_{i=0}^{n} b^{i+1}\left\{\psi_{b}^{i}\left(d_{b}\left(c_{0}, S_{a} c_{0}\right)\right)\right\} \leq r
$$

It follows that

$$
c_{1} \in \overline{B_{d_{b}}\left(c_{0}, r\right)}
$$

Let $c_{2}, \ldots, c_{j} \in \overline{B_{d_{b}}\left(c_{0}, r\right)}$ for some $j \in \mathbb{N}$. If $j$ is odd, then $j=2 \grave{\imath}+1$ for some $i \in \mathbb{N}$. Since $\left\{S_{\sigma}: \sigma \in \Omega\right\}$ and $\left\{T_{\beta}: \beta \in \Phi\right\}$ are two families of $\alpha_{*}$-dominated multivalued mappings on $\overline{B_{d_{b}}\left(c_{0}, r\right)}$, so $\alpha_{*}\left(c_{2 i}, S_{\sigma} c_{2 i}\right) \geq 1$ and $\alpha_{*}\left(c_{2 i+1}, T_{\beta} c_{2 i+1}\right) \geq 1$ for all $\sigma \in \Omega$ and $\beta \in \Phi$. As $\alpha_{*}\left(c_{2 i}, S_{\sigma} c_{2 i}\right) \geq 1$, this implies $\inf \left\{\alpha\left(c_{2 i}, b\right): b \in S_{\sigma} c_{2 i}\right\} \geq 1$. Also $c_{2 i+1} \in S_{f} c_{2 i}$ for some $f \in$ $\Omega$, so $\alpha\left(c_{2 i}, c_{2 i+1}\right) \geq 1$. Also $c_{2 i+1} \in T_{g} c_{2 i+1}$ for some $g \in \Phi$. Now, by using Lemma 1.6, we have

$$
\tau+A\left(d_{b}\left(c_{2 i+1}, c_{2 i+2}\right)\right) \leq \tau+A\left(H_{d_{b}}\left(S_{f} c_{2 i}, T_{g} c_{2 i+1}\right)\right) \leq A\left(\psi_{b}\left(D_{(f, g)}\left(c_{2 i}, c_{2 i+1}\right)\right)\right)
$$




$$
\begin{aligned}
\leq & A\left(\psi _ { b } \left(\operatorname { m a x } \left\{d_{b}\left(c_{2 i}, c_{2 i+1}\right), \frac{d_{b}\left(c_{2 i}, c_{2 i+1}\right) \cdot d_{b}\left(c_{2 i+1}, c_{2 i+2}\right)}{a+d_{b}\left(c_{2 i}, c_{2 i+1}\right)},\right.\right.\right. \\
& \left.\left.\left.d_{b}\left(c_{2 i}, c_{2 i+1}\right), d_{b}\left(c_{2 i+1}, c_{2 i+2}\right)\right\}\right)\right) \\
\leq & A\left(\psi_{b}\left(\max \left\{d_{b}\left(c_{2 i}, c_{2 i+1}\right), d_{b}\left(c_{2 i+1}, c_{2 i+2}\right)\right\}\right)\right) .
\end{aligned}
$$

If $\max \left\{d_{b}\left(c_{2 i}, c_{2 i+1}\right), d_{b}\left(c_{2 \grave{i}+1}, c_{2 \grave{i}+2}\right)\right\}=d_{b}\left(c_{2 \grave{i}+1}, c_{2 i+2}\right)$, then

$$
\tau+A\left(d_{b}\left(c_{2 i+1}, c_{2 i+2}\right)\right) \leq A\left(\psi_{b}\left(d_{b}\left(c_{2 i+1}, c_{2 i+2}\right)\right)\right) .
$$

As $\tau>0$ and $A: \mathbb{R}_{+} \rightarrow \mathbb{R}$ is a strictly increasing function, then

$$
d_{b}\left(c_{2 i+1}, c_{2 i+2}\right)<\psi_{b}\left(d_{b}\left(c_{2 i+1}, c_{2 i+2}\right)\right)<b \psi_{b}\left(d_{b}\left(c_{2 i+1}, c_{2 i+2}\right)\right) .
$$

This is a contradiction to the fact that $b \psi_{b}(t)<t$ for all $t>0$. So

$$
\max \left\{d_{b}\left(c_{2 \grave{i}}, c_{2 \grave{i}+1}\right), d_{b}\left(c_{2 \grave{i}+1}, c_{2 \grave{i}+2}\right)\right\}=d_{b}\left(c_{2 \grave{i}}, c_{2 \grave{i}+1}\right) .
$$

Hence, we obtain

$$
d_{b}\left(c_{2 i+1}, c_{2 i+2}\right)<\psi_{b}\left(d_{b}\left(c_{2 i}, c_{2 \grave{i}+1}\right)\right) .
$$

As $\alpha_{*}\left(c_{2 \grave{i}-1}, T_{h} c_{2 \grave{i}-1}\right) \geq 1$ and $c_{2 \grave{i}} \in T_{h} c_{2 \grave{i}-1}$, so $\alpha\left(c_{2 \grave{i}-1}, c_{2 \grave{i}}\right) \geq 1$ where $h \in \Phi$ and $p \in \Omega$. Now, by using Lemma 1.6, we have

$$
\begin{aligned}
\tau+A\left(d_{b}\left(c_{2 i}, c_{2 i+1}\right)\right) \leq & \tau+A\left(H_{d_{b}}\left(T_{h} c_{2 i-1}, S_{p} c_{2 i}\right)\right) \leq A\left(\psi_{b}\left(D_{(h, p)}\left(c_{2 i}, c_{2 i-1}\right)\right)\right) \\
\leq & A\left(\psi _ { b } \left(\operatorname { m a x } \left\{d_{b}\left(c_{2 i}, c_{2 i-1}\right), \frac{d_{b}\left(c_{2 i}, c_{2 i+1}\right) \cdot d_{b}\left(c_{2 i-1}, c_{2 i}\right)}{a+d_{b}\left(c_{2 i}, c_{2 i-1}\right)}\right.\right.\right. \\
& \left.\left.\left.d_{b}\left(c_{2 i}, c_{2 i+1}\right), d_{b}\left(c_{2 i-1}, c_{2 i}\right)\right\}\right)\right) \\
\leq & A\left(\psi_{b}\left(\max \left\{d_{b}\left(c_{2 i}, c_{2 i-1}\right), d_{b}\left(c_{2 i}, c_{2 \grave{i}+1}\right)\right\}\right)\right) .
\end{aligned}
$$

Since $A: \mathbb{R}_{+} \rightarrow \mathbb{R}$ is a strictly increasing function, then we have

$$
d_{b}\left(c_{2 \grave{i}}, c_{2 \grave{i}+1}\right)<\psi_{b}\left(\max \left\{d_{b}\left(c_{2 \grave{i}}, c_{2 \grave{i}-1}\right), d_{b}\left(c_{2 \grave{i}}, c_{2 \grave{i}+1}\right)\right\}\right) .
$$

If $\max \left\{d_{b}\left(c_{2 i}, c_{2 i-1}\right), d_{b}\left(c_{2 i}, c_{2 i+1}\right)\right\}=d_{b}\left(c_{2 i}, c_{2 i+1}\right)$, then

$$
d_{b}\left(c_{2 i}, c_{2 i+1}\right)<\psi_{b}\left(d_{b}\left(c_{2 i}, c_{2 i+1}\right)\right)<b \psi_{b}\left(d_{b}\left(c_{2 i}, c_{2 i+1}\right)\right) .
$$

This is a contradiction to the fact that $b \psi_{b}(t)<t$ for all $t>0$. Hence, we obtain

$$
d_{b}\left(c_{2 i}, c_{2 i+1}\right)<\psi_{b}\left(d_{b}\left(c_{2 i-1}, c_{2 i}\right)\right) .
$$

As $\psi_{b}$ is nondecreasing, it follows

$$
\psi_{b}\left(d_{b}\left(c_{2 i}, c_{2 i+1}\right)\right)<\psi_{b}\left(\psi_{b}\left(d_{b}\left(c_{2 i-1}, c_{2 i}\right)\right)\right) .
$$


By using the above inequality in (2.3), we have

$$
\left.d_{b}\left(c_{2 \grave{i}+1}, c_{2 \grave{i}+2}\right)<\psi_{b}^{2}\left(d_{b}\left(c_{2 \grave{i}-1}, c_{2 \grave{i}}\right)\right)\right) \text {. }
$$

Continuing in this way, we obtain

$$
d_{b}\left(c_{2 \grave{i}+1}, c_{2 i+2}\right)<\psi_{b}^{2 \grave{i}+1}\left(d_{b}\left(c_{0}, c_{1}\right)\right) .
$$

Now, if $j=2 \grave{i}$, where $\grave{i}=1,2, \ldots, \frac{j}{2}$. By using (2.4) and a similar procedure as above, we have

$$
d_{b}\left(c_{2 \grave{i}}, c_{2 \grave{i}+1}\right)<\psi_{b}^{2 \grave{\imath}}\left(d_{b}\left(c_{0}, c_{1}\right)\right) .
$$

Now, by combining (2.5) and (2.6), we get

$$
d_{b}\left(c_{j}, c_{j+1}\right)<\psi_{b}^{j}\left(d_{b}\left(c_{0}, c_{1}\right)\right) \text { for all } j \in \mathbb{N} \text {. }
$$

Now, by using the triangle inequality and by (2.7), we have

$$
\begin{aligned}
d_{b}\left(c_{0}, c_{j+1}\right) & \leq b d_{b}\left(c_{0}, c_{1}\right)+b^{2} d_{b}\left(c_{1}, c_{2}\right)+\cdots+b^{j+1} d_{b}\left(c_{j}, c_{j+1}\right) \\
& <b d_{b}\left(c_{0}, c_{1}\right)+b^{2} \psi_{b}\left(d_{b}\left(c_{0}, c_{1}\right)\right)+\cdots+b^{j+1} \psi_{b}^{j}\left(d_{b}\left(c_{0}, c_{1}\right)\right) \\
& <\sum_{i=0}^{j} b^{i+1}\left\{\psi_{b}^{i}\left(d_{b}\left(c_{0}, c_{1}\right)\right)\right\}<r .
\end{aligned}
$$

Thus $c_{j+1} \in \overline{B_{d_{b}}\left(c_{0}, r\right)}$. Hence $c_{n} \in \overline{B_{d_{b}}\left(c_{0}, r\right)}$ for all $n \in \mathbb{N}$, therefore $\left\{T_{\beta} S_{\sigma}\left(c_{n}\right)\right\}$ is a sequence in $\overline{B_{d_{b}}\left(c_{0}, r\right)}$. As $S_{\sigma}, T_{\beta}$ are $\alpha_{*}$-dominated on $\overline{B_{d_{b}}\left(c_{0}, r\right)}$, so $\alpha_{*}\left(c_{2 n}, S_{\sigma} c_{2 n}\right) \geq 1$ and $\alpha_{*}\left(c_{2 n+1}, T_{\beta} c_{2 n+1}\right) \geq 1$. This implies $\alpha\left(c_{n}, c_{n+1}\right) \geq 1$. Also inequality (2.7) can be written as

$$
d_{b}\left(c_{n}, c_{n+1}\right)<\psi_{b}^{n}\left(d_{b}\left(c_{0}, c_{1}\right)\right) \text { for all } n \in \mathbb{N} \text {. }
$$

As $\sum_{k=1}^{+\infty} b^{k} \psi_{b}^{k}(t)<+\infty$, then for some $p \in \mathbb{N}$ the series $\sum_{k=1}^{+\infty} b^{k} \psi_{b}^{k}\left(\psi_{b}^{p-1}\left(d_{b}\left(c_{0}, c_{1}\right)\right)\right)$ converges. As $b \psi_{b}(t)<t$, so

$$
b^{n+1} \psi_{b}^{n+1}\left(\psi_{b}^{p-1}\left(d_{b}\left(c_{0}, c_{1}\right)\right)\right)<b^{n} \psi_{b}^{n}\left(\psi_{b}^{p-1}\left(d_{b}\left(c_{0}, c_{1}\right)\right)\right) \quad \text { for all } n \in \mathbb{N} \text {. }
$$

Fix $\varepsilon>0$, then there exists $p(\varepsilon) \in \mathbb{N}$ such that

$$
b \psi_{b}\left(\psi_{b}^{p(\varepsilon)-1}\left(d_{b}\left(c_{0}, c_{1}\right)\right)\right)+b^{2} \psi_{b}^{2}\left(\psi_{b}^{p(\varepsilon)-1}\left(d_{b}\left(c_{0}, c_{1}\right)\right)\right)+\cdots<\varepsilon .
$$

Let $n, m \in \mathbb{N}$ with $m>n>p(\varepsilon)$, then we have

$$
\begin{aligned}
d_{b}\left(c_{n}, c_{m}\right) & \leq b d_{b}\left(c_{n}, c_{n+1}\right)+b^{2} d_{b}\left(c_{n+1}, c_{n+2}\right)+\cdots+b^{m-n} d_{b}\left(c_{m-1}, c_{m}\right) \\
& <b \psi_{b}^{n}\left(d_{b}\left(c_{0}, c_{1}\right)\right)+b^{2} \psi_{b}^{n+1}\left(d_{b}\left(c_{0}, c_{1}\right)\right)+\cdots+b^{m-n} \psi_{b}^{m-1}\left(d_{b}\left(c_{0}, c_{1}\right)\right) \\
& =b \psi_{b}\left(\psi_{b}^{n-1}\left(d_{b}\left(c_{0}, c_{1}\right)\right)\right)+\cdots+b^{m-n} \psi_{b}^{m-n}\left(\psi_{b}^{n-1}\left(d_{b}\left(c_{0}, c_{1}\right)\right)\right) \\
& <b \psi_{b}\left(\psi_{b}^{p(\varepsilon)-1}\left(d_{b}\left(c_{0}, c_{1}\right)\right)\right)+b^{2} \psi_{b}^{2}\left(\psi_{b}^{p(\varepsilon)-1}\left(d_{b}\left(c_{0}, c_{1}\right)\right)\right)+\cdots<\varepsilon .
\end{aligned}
$$


Thus we proved that $\left\{T_{\beta} S_{\sigma}\left(c_{n}\right)\right\}$ is a Cauchy sequence in $\overline{B_{d_{b}}\left(c_{0}, r\right)}$. As $(X, d)$ is complete and $\overline{B_{d_{b}}\left(c_{0}, r\right)}$ is closed, so $\left(\overline{B_{d_{b}}\left(c_{0}, r\right)}, d_{b}\right)$ is complete. This implies that there exist $u \in \overline{B_{d_{b}}\left(c_{0}, r\right)}$ such that $\left\{T_{\beta} S_{\sigma}\left(c_{n}\right)\right\} \rightarrow u$ as $n \rightarrow \infty$, then

$$
\lim _{n \rightarrow \infty} d_{b}\left(c_{n}, u\right)=0
$$

By assumption, $\alpha\left(c_{n}, u\right) \geq 1$. Suppose that $d_{b}\left(u, T_{\beta} u\right)>0$, then there exists a positive integer $k$ such that $d_{b}\left(c_{n}, T_{\beta} u\right)>0$ for all $n \geq k$. For $n \geq k$, we have

$$
d_{b}\left(u, T_{\beta} u\right) \leq b d_{b}\left(u, c_{2 n+1}\right)+b d_{b}\left(c_{2 n+1}, T_{\beta} u\right) .
$$

Now, there exists some $e \in \Omega$ such that $c_{2 n+1} \in S_{e} c_{2 n}$ and $d_{b}\left(c_{2 n}, S_{e} c_{2 n}\right)=d_{b}\left(c_{2 n}, c_{2 n+1}\right)$. By using Lemma 1.6 and inequality (2.1), we have

$$
\begin{aligned}
d_{b}\left(u, T_{\beta} u\right) \leq & b d_{b}\left(u, c_{2 n+1}\right)+b H_{d_{b}}\left(S_{e} c_{2 n}, T_{\beta} u\right), \quad \text { for some } \beta \in \Phi \\
< & b d_{b}\left(u, c_{2 n+1}\right)+b \psi_{b}\left(\operatorname { m a x } \left\{d_{b}\left(c_{2 n}, u\right), d_{b}\left(c_{2 n}, S_{e} c_{2 n}\right),\right.\right. \\
& \left.\left.\frac{d_{b}\left(c_{2 n}, S_{e} c_{2 n}\right) \cdot d_{b}\left(u, T_{\beta} u\right)}{a+d_{b}\left(c_{2 n}, u\right)}, d_{b}\left(u, T_{\beta} u\right)\right\}\right) .
\end{aligned}
$$

Letting $n \rightarrow \infty$, and by using (2.9), we get

$$
d_{b}\left(u, T_{\beta} u\right)<b \psi_{b}\left(d_{b}\left(u, T_{\beta} u\right)\right)<d_{b}\left(u, T_{\beta} u\right),
$$

which is a contradiction. So our supposition is wrong. Hence $d_{b}\left(u, T_{\beta} u\right)=0$ or $u \in T_{\beta} u$ for all $\beta \in \Phi$. Similarly, by using Lemma 1.6 and inequality (2.1), we can show that $d_{b}\left(u, S_{\sigma} u\right)=$ 0 or $u \in S_{\sigma} u$ for all $\sigma \in \Omega$. Hence $S_{\sigma}$ and $T_{\beta}$ have a common fixed point $u$ in $\overline{B_{d_{b}}\left(c_{0}, r\right)}$ for all $\sigma \in \Omega$ and $\beta \in \Phi$. Now,

$$
d_{b}(u, u) \leq b d_{b}\left(u, T_{\beta} u\right)+b d_{b}\left(T_{\beta} u, u\right) \leq 0 .
$$

This implies that $d_{b}(u, u)=0$.

Example 2.2 Let $M=\mathbb{Q}^{+} \cup\{0\}$ and let $d_{b}: M \times M \rightarrow M$ be the complete D.B.M space defined by

$$
d_{b}(\grave{l}, j)=(\grave{i}+j)^{2} \quad \text { for all } i, j \in M
$$

with coefficient $b=2$. Define $S_{\sigma}, T_{\beta}: M \times M \rightarrow P(M)$ to be two families of multivalued mappings by

$$
S_{m} x=\left\{\begin{array}{ll}
{\left[\frac{x}{3 m}, \frac{2}{3 m} x\right]} & \text { if } x \in[0,14] \cap M, \\
{[x m, 2 m x]} & \text { if } x \in(14, \infty) \cap M
\end{array} \quad \text { where } m=1,2,3, \ldots\right.
$$

and

$$
T_{n} x=\left\{\begin{array}{ll}
{\left[\frac{x}{4 n}, \frac{3}{4 n} x\right]} & \text { if } x \in[0,14] \cap M, \\
{[2 n x, 3 n x]} & \text { if } x \in(14, \infty) \cap M .
\end{array} \quad \text { where } n=1,2,3, \ldots\right.
$$


Suppose that, $x_{0}=1, r=225, a=1$, then $\overline{B_{d_{b}}\left(x_{0}, r\right)}=[0,14] \cap M$. Now, $d_{b}\left(x_{0}, S_{1} x_{0}\right)=$ $d_{b}\left(1, S_{1} 1\right)=d_{b}\left(1, \frac{1}{3}\right)$. So $x_{1}=\frac{1}{3}$. Now, $d_{b}\left(x_{1}, T_{1} x_{1}\right)=d_{b}\left(\frac{1}{3}, T_{1} \frac{1}{3}\right)=d_{b}\left(\frac{1}{3}, \frac{1}{12}\right)$. So $x_{2}=\frac{1}{12}$. Now, $d_{b}\left(x_{2}, S_{2} x_{2}\right)=d_{b}\left(\frac{1}{12}, S_{2} \frac{1}{12}\right)=d_{b}\left(\frac{1}{12}, \frac{1}{72}\right)$. So $x_{3}=\frac{1}{72}$. Continuing in this way, we have $\left\{T_{n} S_{m}\left(x_{n}\right)\right\}=\left\{1, \frac{1}{3}, \frac{1}{12}, \frac{1}{72}, \ldots\right\}$. Let $\psi_{b}(t)=\frac{4 t}{10}$, then $b \psi_{b}(t)<t$. Consider the mapping $\alpha$ : $M \times M \rightarrow[0, \infty)$ by

$$
\alpha(j, k)=\left\{\begin{array}{ll}
1 & \text { if } j>k \\
\frac{1}{2} & \text { otherwise }
\end{array}\right\} .
$$

Now, if $x, y \in \overline{B_{d_{b}}\left(x_{0}, r\right)} \cap\left\{T_{\beta} S_{\sigma}\left(x_{n}\right)\right\}$ with $\alpha(x, y) \geq 1$, we have

$$
\begin{aligned}
H_{d_{b}}\left(S_{m} x, T_{n} y\right)= & \max \left\{\sup _{a \in S_{m} x} d_{b}\left(a, T_{n} y\right), \sup _{b \in T_{n} y} d_{b}\left(S_{m} x, b\right)\right\} \\
= & \max \left\{\sup _{a \in S_{m} x} d_{b}\left(a,\left[\frac{y}{4 n}, \frac{3 y}{4 n}\right]\right), \sup _{b \in T_{n} y} d_{b}\left(\left[\frac{x}{3 m}, \frac{2 x}{3 m}\right], b\right)\right\} \\
= & \max \left\{d_{b}\left(\frac{2 x}{3 m},\left[\frac{y}{4 n}, \frac{3 y}{4 n}\right]\right), d_{b}\left(\left[\frac{x}{3 m}, \frac{2 x}{3 m}\right], \frac{3 y}{4 n}\right)\right\} \\
= & \max \left\{d_{b}\left(\frac{2 x}{3 m}, \frac{y}{4 n}\right), d_{b}\left(\frac{x}{3 m}, \frac{3 y}{4 n}\right)\right\} \\
= & \max \left\{\left(\frac{2 x}{3 m}+\frac{y}{4 n}\right)^{2},\left(\frac{x}{3 m}+\frac{3 y}{4 n}\right)^{2}\right\} \\
& <\psi_{b}\left\{\operatorname { m a x } \left((x+y)^{2}, \frac{\left(x+\frac{x}{3 m}\right)^{4} \cdot\left(y+\frac{y}{4 n}\right)^{2}}{\left\{1+(x+y)^{4}\right\}},\right.\right. \\
& \left.\left.\left(x+\frac{x}{3 m}\right)^{2},\left(x+\frac{y}{4 n}\right)^{2}\right)\right\} \\
& <\psi_{b}\left\{\operatorname { m a x } \left(d_{b}(x, y), \frac{d_{b}\left(x,\left[\frac{x}{3 m}, \frac{2}{3 m} x\right]\right) \cdot d_{b}\left(y,\left[\frac{y}{4 n}, \frac{3}{4 n} y\right]\right)}{1+d_{b}(x, y)}\right.\right. \\
& \left.\left.d_{b}\left(x,\left[\frac{x}{3 m}, \frac{2}{3 m} x\right]\right), d_{b}\left(x,\left[\frac{y}{4 n}, \frac{3}{4 n} y\right]\right)\right)\right\} .
\end{aligned}
$$

Thus,

$$
\left.H_{d_{b}}(S x, T y)\right)<\psi_{b}\left(D_{(\sigma, \beta)}(x, y)\right)
$$

which implies that, for any $\tau \in\left(0, \frac{12}{95}\right]$ and for a strictly increasing mapping $A(s)=\ln s$, we have

$$
\tau+A\left(H_{d_{l}}\left(S_{m} x, T_{n} y\right)\right) \leq A\left(\psi_{b}\left(D_{(\sigma, \beta)}(x, y)\right)\right) .
$$

Note that, for $15,16 \in M$, then $\alpha(16,15) \geq 1$. However, we have

$$
\tau+A\left(H_{d_{l}}\left(S_{2} 16, T_{1} 15\right)\right)>A\left(\psi_{b}\left(D_{(\sigma, \beta)}(16,15)\right)\right) .
$$


So condition (2.1) does not hold on $M$. Also, for all $n \in \mathbb{N} \cup\{0\}$, we have

$$
\sum_{i=0}^{n} b^{i+1}\left\{\psi_{b}^{i}\left(d_{b}\left(x_{0}, S_{1} x_{0}\right)\right)\right\}=\frac{16}{9} \times 2 \sum_{i=0}^{n}\left(\frac{4}{5}\right)^{i}<225=r .
$$

Thus all the conditions of Theorem 2.1 are satisfied. Hence $S_{\sigma}$ and $T_{\beta}$ have a common fixed point for all $\sigma \in \Omega$ and $\beta \in \Phi$, that is, 0 .

Corollary 2.3 Let $\left(M, d_{b}\right)$ be a complete D.B.M space. Suppose that there exists a function $\alpha: M \times M \rightarrow[0, \infty)$. Let $r>0, c_{0} \in \overline{B_{d_{b}}\left(c_{0}, r\right)}$, A be a strictly increasing function, and $\left\{S_{\sigma}: \sigma \in \Omega\right\},\left\{T_{\beta}: \beta \in \Phi\right\}$ be two families of $\alpha_{*}$-dominated self mappings from $M$ to $M$ on $\overline{B_{d_{b}}\left(c_{0}, r\right)}$. Suppose that, for some $\psi_{b} \in \Psi_{b}$, there exists $\tau>0$ such that the following holds:

$$
\tau+A\left(H_{d_{b}}\left(S_{\sigma} e, T_{\beta} y\right)\right) \leq A\left(\psi_{b}\left(D_{(\sigma, \beta)}(e, y)\right)\right)
$$

for all $e, y \in \overline{B_{d_{b}}\left(c_{0}, r\right)} \cap\left\{T_{\beta} S_{\sigma}\left(c_{n}\right)\right\}, \alpha(e, y) \geq 1, \sigma \in \Omega, \beta \in \Phi$, and $H_{d_{b}}\left(S_{\sigma} e, T_{\beta} y\right)>0$. Also

$$
\sum_{i=0}^{n} b^{i+1}\left\{\psi_{b}^{i}\left(d_{b}\left(c_{0}, S_{a} c_{0}\right)\right)\right\} \leq r \quad \text { for all } n \in \mathbb{N} \cup\{0\}
$$

Then $\left\{T_{\beta} S_{\sigma}\left(c_{n}\right)\right\}$ is a sequence in $\overline{B_{d_{b}}\left(c_{0}, r\right)}, \alpha\left(c_{n}, c_{n+1}\right) \geq 1$ for all $n \in \mathbb{N} \cup\{0\}$ and $\left\{T_{\beta} S_{\sigma}\left(c_{n}\right)\right\} \rightarrow u \in \overline{B_{d_{b}}\left(c_{0}, r\right)}$. Also, $u$ satisfies (2.10), if $\overline{B_{d_{b}}\left(c_{0}, r\right)}$ is a closed set and either $\alpha\left(c_{n}, u\right) \geq 1$ or $\alpha\left(u, c_{n}\right) \geq 1$ for all $n \in \mathbb{N} \cup\{0\}$, then $S_{\sigma}$ and $T_{\beta}$ have a common fixed point $u$ in $\overline{B_{d_{b}}\left(c_{0}, r\right)}$ for all $\sigma \in \Omega$ and $\beta \in \Phi$.

Corollary 2.4 Let $\left(M, d_{b}\right)$ be a complete D.B.M space. Suppose that there exists a function $\alpha: M \times M \rightarrow[0, \infty)$. Let $r>0, c_{0} \in \overline{B_{d_{b}}\left(c_{0}, r\right)}, A$ be a strictly increasing function, and $\left\{S_{\sigma}:\right.$ $\sigma \in \Omega\}$ be the family of $\alpha_{*}$-dominated multivalued mappings from $M$ to $P(M)$ on $\overline{B_{d_{b}}\left(c_{0}, r\right)}$. Suppose that, for some $\psi_{b} \in \Psi_{b}$, there exists $\tau>0$ such that the following holds:

$$
\tau+A\left(H_{d_{b}}\left(S_{\sigma} e, S_{\beta} y\right)\right) \leq A\left(\psi_{b}\left(D_{(\sigma, \beta)}(e, y)\right)\right)
$$

for all $e, y \in \overline{B_{d_{b}}\left(c_{0}, r\right)} \cap\left\{M S_{\sigma}\left(c_{n}\right)\right\}, \alpha(e, y) \geq 1, \sigma, \beta \in \Omega$, and $H_{d_{b}}\left(S_{\sigma} e, S_{\beta} y\right)>0$. Also

$$
\sum_{i=0}^{n} b^{i+1}\left\{\psi_{b}^{i}\left(d_{b}\left(c_{0}, S_{a} c_{0}\right)\right)\right\} \leq r \quad \text { for all } n \in \mathbb{N} \cup\{0\}
$$

Then $\left\{M S_{\sigma}\left(c_{n}\right)\right\}$ is a sequence in $\overline{B_{d_{b}}\left(c_{0}, r\right)}, \alpha\left(c_{n}, c_{n+1}\right) \geq 1$ for all $n \in \mathbb{N} \cup\{0\}$ and $\left\{M S_{\sigma}\left(c_{n}\right)\right\} \rightarrow u \in \overline{B_{d_{b}}\left(c_{0}, r\right)}$. Also, $u$ satisfies (2.11), if $\overline{B_{d_{b}}\left(c_{0}, r\right)}$ is a closed set and either $\alpha\left(c_{n}, u\right) \geq 1$ or $\alpha\left(u, c_{n}\right) \geq 1$ for all $n \in \mathbb{N} \cup\{0\}$, then $S_{\sigma}$ has a common fixed point $u$ in $\overline{B_{d_{b}}\left(c_{0}, r\right)}$ for all $\sigma \in \Omega$.

\section{Results for families of multigraph dominated mappings}

In this section we present an application of Theorem 2.1 in graph theory. Jachymski [16] proved the result concerning for contraction mappings on metric space with a graph. Hussain et al. [13], introduced the fixed points theorem for graphic contraction and gave an application. 
Definition 3.1 Let $X$ be a nonempty set and $G=(V(G), E(G))$ be a graph such that $V(G)=$ $X, A \subseteq X$. A mapping $F: X \rightarrow P(X)$ is said to be multigraph dominated on $A$ if $(x, y) \in E(G)$ for all $y \in F x$ and $x \in A$.

Theorem 3.2 Let $\left(M, d_{b}\right)$ be a complete D.B.M space endowed with a graph $G$ with constant $b \geq 1$. Let $r>0, c_{0} \in \overline{B_{d_{b}}\left(c_{0}, r\right)}$, and $\left\{S_{\sigma}: \sigma \in \Omega\right\},\left\{T_{\beta}: \beta \in \Phi\right\}$ be two families of multivalued mappings from $M$ to $P(M)$. Suppose that

(i) $\left\{S_{\sigma}: \sigma \in \Omega\right\},\left\{T_{\beta}: \beta \in \Phi\right\}$ are two families of multigraph dominated on $\overline{B_{d_{b}}\left(c_{0}, r\right)} \cap\left\{T_{\beta} S_{\sigma}\left(c_{n}\right)\right\}$.

(ii) There exist $\tau>0$ and a strictly increasing mapping $A$ satisfying

$$
\tau+A\left(H_{d_{b}}\left(S_{\sigma} e, T_{\beta} y\right)\right) \leq A\left(\psi_{b}\left(D_{(\sigma, \beta)}(e, y)\right)\right)
$$

whenever e, $y \in \overline{B_{d_{b}}\left(c_{0}, r\right)} \cap\left\{T_{\beta} S_{\sigma}\left(c_{n}\right)\right\},(e, y) \in E(G), \sigma \in \Omega, \beta \in \Phi$, and $H_{d_{b}}\left(S_{\sigma} e, T_{\beta} y\right)>0$.

(iii) $\sum_{i=0}^{n} b^{i+1}\left\{\psi_{b}^{i}\left(d_{b}\left(x_{0}, S_{\sigma} c_{0}\right)\right)\right\} \leq$ for all $n \in N$.

Then $\left\{T_{\beta} S_{\sigma}\left(c_{n}\right)\right\}$ is a sequence in $\overline{B_{d_{b}}\left(c_{0}, r\right)}$ if $\overline{B_{d_{b}}\left(c_{0}, r\right)}$ is a closed set $\left(c_{n}, c_{n+1}\right) \in E(G)$ and $\left\{T_{\beta} S_{\sigma}\left(c_{n}\right)\right\} \rightarrow m^{*}$. Also, if $m^{*}$ satisfies (3.1) and $\left(c_{n}, m^{*}\right) \in E(G)$ or $\left(m^{*}, c_{n}\right) \in E(G)$ for all $n \in N \cup\{0\}$, then $S_{\sigma}$ and $T_{\beta}$ have a common fixed point $m^{*}$ in $\overline{B_{d_{b}}\left(c_{0}, r\right)}$ for all $\sigma \in \Omega$ and $\beta \in \Phi$.

Proof Define $\alpha: M \times M \rightarrow[0, \infty)$ by

$$
\alpha(e, y)= \begin{cases}1 & \text { if } e \in \overline{B_{d_{b}}\left(c_{0}, r\right)},(e, y) \in E(G) \\ 0 & \text { otherwise }\end{cases}
$$

As $S_{\sigma}$ and $T_{\beta}$ are two families of graphs dominated on $\overline{B_{d_{b}}\left(c_{0}, r\right)}$, then for $e \in \overline{B_{d_{b}}\left(c_{0}, r\right)}$, $(e, y) \in E(G)$ for all $y \in S_{\sigma} e$ and $(e, y) \in E(G)$ for all $y \in T_{\beta} e$. So, $\alpha(e, y)=1$ for all $y \in S_{\sigma} e$ and $\alpha(e, y)=1$ for all $y \in T_{\beta} e$. This implies that $\inf \left\{\alpha(e, y): y \in S_{\sigma} e\right\}=1$ and $\inf \{\alpha(e, y): y \in$ $\left.T_{\beta} e\right\}=1$. Hence $\alpha_{*}\left(e, S_{\sigma} e\right)=1, \alpha_{*}\left(e, T_{\beta} e\right)=1$ for all $e \in \overline{B_{d_{b}}\left(c_{0}, r\right)}$. So, $S_{\sigma}, T_{\beta}: M \rightarrow P(M)$ are two families of $\alpha_{*}$-dominated mappings on $\overline{B_{d_{b}}\left(c_{0}, r\right)}$. Moreover, inequality (3.1) can be written as

$$
\tau+A\left(H_{d_{b}}\left(S_{\sigma} e, T_{\beta} y\right)\right) \leq A\left(\psi_{b}\left(D_{(\sigma, \beta)}(e, y)\right)\right)
$$

whenever $e, y \in \overline{B_{d_{b}}\left(c_{0}, r\right)} \cap\left\{T_{\beta} S_{\sigma}\left(c_{n}\right)\right\}, \alpha(e, y) \geq 1$, and $H_{d_{b}}\left(S_{\sigma} e, T_{\beta} y\right)>0$. Also, (iii) holds. Then, by Theorem 2.1, we have $\left\{T_{\beta} S_{\sigma}\left(c_{n}\right)\right\}$ is a sequence in $\overline{B_{d_{b}}\left(c_{0}, r\right)}$ and $\left\{T_{\beta} S_{\sigma}\left(c_{n}\right)\right\} \rightarrow$ $m^{*} \in \overline{B_{d_{b}}\left(c_{0}, r\right)}$. Now, $c_{n}, m^{*} \in \overline{B_{d_{b}}\left(c_{0}, r\right)}$ and either $\left(c_{n}, m^{*}\right) \in E(G)$ or $\left(m^{*}, c_{n}\right) \in E(G)$ implies that either $\alpha\left(c_{n}, m^{*}\right) \geq 1$ or $\alpha\left(m^{*}, c_{n}\right) \geq 1$. So, all the conditions of Theorem 2.1 are satisfied. Hence, by Theorem 2.1, $S_{\sigma}$ and $T_{\beta}$ have a common fixed point $m^{*}$ in $\overline{B_{d_{b}}\left(c_{0}, r\right)}$ and $d_{b}\left(m^{*}, m^{*}\right)=0$.

\section{Application to the systems of integral equations}

Theorem 4.1 Let $\left(M, d_{b}\right)$ be a complete D.B.M space with coefficient $b \geq 1$. Let $c_{0} \in M$ and $\left\{S_{\sigma}: \sigma \in \Omega\right\},\left\{T_{\beta}: \beta \in \Phi\right\}$ be two families of mappings from $M$ to $M$. Assume that 
there exists $\tau>0$ and $A: \mathbb{R}_{+} \rightarrow \mathbb{R}$ is a strictly increasing mapping such that the following holds:

$$
\tau+A\left(d_{b}\left(S_{\sigma} e, T_{\beta} y\right)\right) \leq A\left(\psi_{b}\left(D_{(\sigma, \beta)}(e, y)\right)\right)
$$

whenever $e, y \in\left\{T_{\beta} S_{\sigma}\left(c_{n}\right)\right\}, \sigma \in \Omega, \beta \in \Phi$, and $d_{b}\left(S_{\sigma} e, T_{\beta} y\right)>0$. Then $\left\{T_{\beta} S_{\sigma}\left(c_{n}\right)\right\} \rightarrow u \in M$. Also, if inequality (4.1) holds for $e, y \in\{u\}$, then $S_{\sigma}$ and $T_{\beta}$ have a unique common fixed point $u$ in $M$ for all $\sigma \in \Omega$ and $\beta \in \Phi$.

Proof The proof of this theorem is similar as that of Theorem 2.1. We have to prove the uniqueness only. Let $v$ be another common fixed point of $S_{\sigma}$ and $T_{\beta}$. Suppose $d_{b}\left(S_{\sigma} u, T_{\beta} v\right)>0$. Then we have

$$
\tau+A\left(d_{b}\left(S_{\sigma} u, T_{\beta} v\right)\right) \leq A\left(\psi_{b}\left(D_{(\sigma, \beta)}(u, v)\right)\right)
$$

This implies that

$$
d_{b}(u, v)<\psi_{b} d_{b}(u, v)<b \psi_{b} d_{b}(u, v)<d_{b}(u, v),
$$

which is a contradiction. So $d_{b}\left(S_{\sigma} u, T_{\beta} v\right)=0$. Hence $u=v$.

In this section, we discuss the application of fixed point Theorem 4.1 in a form of unique solution of two families of Volterra type integral equations given below:

$$
\begin{aligned}
& u(k)=\int_{0}^{k} H_{\sigma}(k, h, u) d h, \\
& \left.c(k)=\int_{0}^{k} G_{\beta}(k, h, c)\right) d h
\end{aligned}
$$

for all $k \in[0,1], \sigma \in \Omega, \beta \in \Phi$, and $H_{\sigma}, G_{\beta}$ be the mappings from $[0,1] \times[0,1] \times$ $C\left([0,1], \mathbb{R}_{+}\right)$to $\mathbb{R}$. We find the solution of (4.2) and (4.3). Let $M=C\left([0,1], \mathbb{R}_{+}\right)$be the set of all continuous functions on $[0,1]$ with nonnegative values endowed with the complete dislocated $b$-metric. For $u \in C\left([0,1], \mathbb{R}_{+}\right)$, define supremum norm as $\|u\|_{\tau}=$ $\sup _{k \in[0,1]}\left\{|u(k)| e^{-\tau k}\right\}$, where $\tau>0$ is taken arbitrarily. Then define

$$
d_{\tau}(u, c)=\left[\sup _{k \in[0,1]}\left\{|u(k)+c(k)| e^{-\tau k}\right\}\right]^{2}=\|u+c\|_{\tau}^{2}
$$

for all $u, c \in C\left([0,1], \mathbb{R}_{+}\right)$, with these settings, $\left(C\left([0,1], \mathbb{R}_{+}\right), d_{\tau}\right)$ becomes a complete $D . B . M$ space.

Now we prove the following theorem to ensure the existence of solution of integral equations.

Theorem 4.2 Assume that the following conditions are satisfied:

(i) $\left\{H_{\sigma}, \sigma \in \Omega\right\},\left\{G_{\beta}, \beta \in \Phi\right\}$ are two families of mappings from $[0,1] \times[0,1] \times C\left([0,1], \mathbb{R}_{+}\right)$to $\mathbb{R}$; 
(ii) Define

$$
\begin{aligned}
& \left(S_{\sigma} u\right)(k)=\int_{0}^{k} H_{\sigma}(k, h, u) d h, \\
& \left(T_{\beta} c\right)(k)=\int_{0}^{k} G_{\beta}(k, h, c) d h .
\end{aligned}
$$

Suppose that there exists $\tau>0$ such that

$$
\left|H_{\sigma}(k, h, u)+G_{\beta}(k, h, c)\right| \leq \frac{\tau D_{(\sigma, \beta)}(u, c)}{\tau D_{(\sigma, \beta)}(u, c)+1}
$$

for all $k, h \in[0,1]$ and $u, c \in C([0,1], \mathbb{R})$, where

$$
\begin{aligned}
D_{(\sigma, \beta)}(u, c)= & \max \left\{\psi _ { b } \left(\|u+c\|_{\tau}^{2}, \frac{\left\|u+S_{\sigma} u\right\|_{\tau}^{2} \cdot\left\|c+T_{\beta} c\right\|_{\tau}^{2}}{1+\|u(h)+c(h)\|_{\tau}^{2}},\right.\right. \\
& \left.\left.\left\|u+S_{\sigma} u\right\|_{\tau}^{2},\left\|c+T_{\beta} c\right\|_{\tau}^{2}\right)\right\} .
\end{aligned}
$$

Then integral Eqs. (4.2) and (4.3) have a unique solution in $C\left([0,1], \mathbb{R}_{+}\right)$.

Proof By assumption (ii)

$$
\begin{aligned}
\left|S_{\sigma} u+T_{\beta} c\right| & =\int_{0}^{k}\left|H_{\sigma}(k, h, u)+G_{\beta}(k, h, c)\right| d h \\
& \leq \int_{0}^{k} \frac{\tau D_{(\sigma, \beta)}(u, c)}{\tau D_{(\sigma, \beta)}(u, c)+1} e^{\tau h} d h \\
& \leq \frac{\tau D_{(\sigma, \beta)}(u, c)}{\tau D_{(\sigma, \beta)}(u, c)+1} \int_{0}^{k} e^{\tau h} d h \\
& \leq \frac{D_{(\sigma, \beta)}(u, c)}{\tau D_{(\sigma, \beta)}(u, c)+1} e^{\tau k} .
\end{aligned}
$$

This implies

$$
\begin{aligned}
& \left|S_{\sigma} u+T_{\beta} c\right| e^{-\tau k} \leq \frac{D_{(\sigma, \beta)}(u, c)}{\tau D_{(\sigma, \beta)}(u, c)+1} . \\
& \left\|S_{\sigma} u+T_{\beta} c\right\|_{\tau} \leq \frac{D_{(\sigma, \beta)}(u, c)}{\tau D_{(\sigma, \beta)}(u, c)+1} . \\
& \frac{\tau D_{(\sigma, \beta)}(u, c)+1}{D_{(\sigma, \beta)}(u, c)} \leq \frac{1}{\left\|S_{\sigma} u+T_{\beta} c\right\|_{\tau}} . \\
& \tau+\frac{1}{D_{(\sigma, \beta)}(u, c)} \leq \frac{1}{\left\|S_{\sigma} u+T_{\beta} c\right\|_{\tau}},
\end{aligned}
$$

which further implies

$$
\tau-\frac{1}{\left\|S_{\sigma} u+T_{\beta} c\right\|_{\tau}} \leq \frac{-1}{D_{(\sigma, \beta)}(u, c)} .
$$


So all the conditions of Theorem 4.1 are satisfied for $A(c)=\frac{-1}{\sqrt{c}} ; c>0$ and $d_{\tau}(u, c)=\|u+c\|_{\tau}^{2}$. Hence two families of integral equations given in (4.2) and (4.3) have a unique common solution.

Example 4.3 Consider the integral equations

$$
\left.g(k)=\frac{1}{5} \int_{0}^{k} g(h) d h, \quad p(k)=\frac{1}{7} \int_{0}^{k} p(h)\right) d h, \quad \text { where } k \in[0,1] .
$$

Define $\left\{H_{\sigma}, \sigma \in \Omega\right\},\left\{H_{\beta}, \beta \in \Phi\right\}$ to be two families of mappings from $[0,1] \times[0,1] \times$ $C\left([0,1], \mathbb{R}_{+}\right)$to $\mathbb{R}$; by $H_{\sigma}=\frac{1}{5} g(h), H_{\beta}=\frac{1}{7} p(h)$. Now,

$$
\left.\left(S_{\sigma} g\right)(k)=\frac{1}{5} \int_{0}^{k} g(h) d h, \quad\left(T_{\beta} p\right)(k)=\frac{1}{7} \int_{0}^{k} p(h)\right) d h .
$$

Take $\tau=\frac{6}{97},\|u\|_{\tau}=\sup _{k \in[0,1]}\left\{|u(k)| e^{-\tau k}\right\}$ and $\psi_{b}(t)=\frac{4 t}{10}$. Then all the conditions of Theorem 4.2 are satisfied and $g(k)=p(k)=0$ for all $k$ is a unique common solution to the above equations.

\section{Conclusion}

In the present paper, we achieved fixed point results for a pair of families of multivalued generalized $\alpha_{*}-\psi$-dominated contractive mappings on an intersection of a closed ball and a sequence for a more general class of $\alpha_{*}$-dominated mappings rather than $\alpha_{*}$ admissible mappings and for a weaker class of strictly increasing mappings $A$ rather than the class of mappings $F$ used by Wardowski [29]. The notion of multigraph dominated mapping is introduced. Fixed point results with graphic contractions on a closed ball for such families of mappings are established. Examples are given to demonstrate the variety of our results. An application is given to approximate the unique common solution of two families of nonlinear integral equations. Moreover, we investigated our results in a better new framework. New results in ordered spaces, partial $b$-metric space, dislocated metric space, partial metric space, $b$-metric space, and metric space can be obtained as corollaries of our results.

\section{Acknowledgements}

This article was supported by the Deanship of Scientific Research (DSR), King Abdulaziz University, Jeddah. Article processing charge will be given by DSR, King Abdulaziz University. Therefore, the authors acknowledge with thanks DSR, KAU, for financial support.

\section{Funding}

This project was funded by the Deanship of Scientific Research (DSR) at King Abdulaziz University, Jeddah under grant No. RG-18-130-37.

\section{Competing interests}

The authors declare that they do not have any competing interests.

Authors' contributions

Each author equally contributed to this paper, read and approved the final manuscript.

\section{Author details}

${ }^{1}$ Department of Mathematics and Statistics, International Islamic University, Islamabad, Pakistan. ${ }^{2}$ Department of Mathematics and Statistics, Riphah International University, Islamabad, Pakistan. ${ }^{3}$ Dipartimento di Matematica e Informatica, Universita della Calabria, Arcavacata di Rende (CS), Italy. ${ }^{4}$ Department of Mathematics, King Abdulaziz University, Jeddah, Saudi Arabia. 


\section{Publisher's Note}

Springer Nature remains neutral with regard to jurisdictional claims in published maps and institutional affiliations.

\section{Received: 23 February 2019 Accepted: 7 June 2019 Published online: 28 June 2019}

\section{References}

1. Acar, Ö., Durmaz, G., Minak, G.: Generalized multivalued F-contractions on complete metric spaces. Bull. Iran. Math. Soc. 40, 1469-1478 (2014)

2. Ali, M.U., Kamran, T., Karapınar, E.: Further discussion on modified multivalued $\alpha^{*}-\psi$-contractive type mapping. Filomat 29(8), 1893-1900 (2015)

3. Alofi, A.S.M., Al-Mazrooei, A.E., Leyew, B.T., Abbas, M.: Common fixed points of $\alpha$-dominated multivalued mappings on closed balls in a dislocated quasi b-metric space. J. Nonlinear Sci. Appl. 10(7), 3456-3476 (2017)

4. Ameer, E., Arshad, M.: Two new generalization for F-contraction on closed ball and fixed point theorem with application. J. Math. Ext. 11, 1-24 (2017)

5. Arshad, M., Hussain, A., Azam, A.: Fixed Point of $\alpha$-Geraghty contraction with applications. U.P.B. Bull. Sci. 78(2), 67-78 (2016)

6. Arshad, M., Khan, S.U., Ahmad, J.: Fixed point results for F-contractions involving some new rational expressions. J. Fixed Point Theory Appl. 11(1), 79-97 (2016)

7. Azam, A., Rashid, M., Mehmood, N.: Set-valued $(\Psi, \Phi)-\Theta$ ordered contractions with applications in diferential inclusions. J. Anal. (2018). https://doi.org/10.1007/s41478-018-0107-4

8. Boriceanu, M.: Fixed point theory for multivalued generalized contraction on a set with two $b$-metrics. Stud. Univ. Babeş-Bolyai, Math. 65(3), 1-14 (2009)

9. Bota, M.F., Chifu, C., Karapinar, E.: Fixed point theorem for generalized $\left(\alpha_{*}-\psi\right)$ Ćirić-type contractive multivalued operator in b-metric spaces. J. Nonlinear Sci. Appl. 9, 1165-1177 (2016)

10. Chen, C., Wen, L., Dong, J., Gu, Y.: Fixed point theorems for generalized $F$-contractions in $b$-metric-like spaces. J. Nonlinear Sci. Appl. 9, 2161-2174 (2016)

11. Czerwik, S.: Contraction mappings in b-metric spaces. Acta Math. Inform. Univ. Ostrav. 1, 511 (1993)

12. Hussain, N., Ahmad, J., Azam, A.: Generalized fixed point theorems for multi-valued $\alpha-\psi$-contractive mappings. J. Inequal. Appl. 2014, 348 (2014)

13. Hussain, N., Al-Mezel, S., Salimi, P.: Fixed points for $\psi$-graphic contractions with application to integral equations. Abstr. Appl. Anal. 2013, Article ID 575869 (2013)

14. Hussain, N., Roshan, J.R., Paravench, V., Abbas, M.: Common fixed point results for weak contractive mappings in ordered dislocated b-metric space with applications. J. Inequal. Appl. 2013, 486, 1-21 (2013)

15. Hussain, N., Salimi, P.: Suzuki-Wardowski type fixed point theorems for $\alpha$-GF-contractions. Taiwan. J. Math. 18(6), 1879-1895 (2014). https://doi.org/10.11650/tjm.18.2014.4462

16. Jachymski, J.: The contraction principle for mappings on a metric space with a graph. Proc. Am. Math. Soc. 1(136), 1359-1373 (2008)

17. Mahmood, Q., Shoaib, A., Rasham, T., Arshad, M.: Fixed point results for the family of multivalued F-contractive mappings on closed ball in complete dislocated b-metric spaces. Mathematics 7(1), 56 (2019)

18. Nadler, S.B.: Multivalued contraction mappings. Pac. J. Math. 30, 475-488 (1969)

19. Nazir, T., Silvestrov, S.: Common fixed point results for family of generalized multivalued $F$-contraction mappings in ordered metric spaces, arXiv:1606.05299v1 [math GM], 17 pages

20. Piri, H., Kumam, P.: Some fixed point theorems concerning F-contraction in complete metric spaces. Fixed Point Theory Appl. 2014, 210 (2014)

21. Piri, H., Rahrovi, S., Morasi, H., Kumam, P.: Fixed point theorem for F-Khan-contractions on complete metric spaces and application to the integral equations. J. Nonlinear Sci. Appl. 10, 4564-4573 (2017)

22. Rasham, T., Shoaib, A., Alamri, B.S., Arshad, M.: Multivalued fixed point results for new generalized F-dominated contractive mappings on dislocated metric space with application. J. Funct. Spaces 2018, Article ID 4808764 (2018)

23. Rasham, T., Shoaib, A., Hussain, N., Arshad, M., Khan, S.U.: Common fixed point results for new Ciric-type rational multivalued F-contraction with an application. J. Fixed Point Theory Appl. 20(1), 45, 16 pages (2018)

24. Sgroi, M., Vetro, C.: Multi-valued F-contractions and the solution of certain functional and integral equations. Filomat 27(7), 1259-1268 (2013)

25. Shoaib, A.: Fixed point results for $\alpha_{*}-\psi$-multivalued mappings. Bull. Math. Anal. Appl. 8(4), 43-55 (2016)

26. Shoaib, A., Azam, A., Arshad, M., Shahzad, A.: Fixed point results for the multivalued mapping on closed ball in dislocated fuzzy metric space. J. Math. Anal. 8(2), 98-106 (2017)

27. Shoaib, A., Azam, A., Shahzad, A.: Common fixed point results for the family of multivalued mappings satisfying contraction on a sequence in Hausdorff fuzzy metric space. J. Comput. Anal. Appl. 24(4), 692-699 (2018)

28. Shoaib, A., Hussain, A., Arshad, M., Azam, A.: Fixed point results for $\alpha_{*}-\psi$-Ciric type multivalued mappings on an intersection of a closed ball and a sequence with graph. J. Math. Anal. 7(3), 41-50 (2016)

29. Wardowski, D.: Fixed point theory of a new type of contractive mappings in complete metric spaces. Fixed Point Theory Appl. 2012, Article ID 94 (2012) 